\title{
Numerical Study of the Effect Surface Properties in Boiling
}

\author{
E. Freitas ${ }^{1}$, D. Bento ${ }^{2}$, R. Lima ${ }^{1,2}$, J. M. Miranda ${ }^{2, *}$, A.S. Moita ${ }^{3}$, A.L.N. Moreira ${ }^{3}$ \\ ${ }^{1}$ MEtRiCS, Mechanical Eng. Dep., University of Minho, \\ Campus de Azurém, 4800-058 Guimarães, Portugal. \\ ${ }^{2}$ CEFT, Faculdade de Engenharia da Universidade do Porto (FEUP) \\ Ruas Dr. Roberto Frias, 4200-465 Porto, Portugal. \\ ${ }^{3} \mathrm{IN}+$, Instituto Superior Técnico, Universidade de Lisboa, \\ Av. Rovisco Pais, 1049-001 Lisbon, Portugal \\ *corresponding author: jmiranda@fe.up.pt
}

\begin{abstract}
High heat loads are common to most industries, such as microprocessors, power generators, thermal management and transportation. One solution for this problem is the use of systems combining evaporator, condenser and electrowetting heat pipes. Conventional evaporators can limit the operation of the system if they are limited by heat conduction in a film near the wall surface. To improve the system modification on the surface wetting have been proposed, through change in the surface properties (contact angle) or through electrowetting. In this work, a numerical simulation of boiling in different wetting conditions is presented. The two-phase flow is described by the VOF method. Integrated heat transport of both phases is solved. The evolution of bubble shape and the temperature field during bubble formation are analysed.
\end{abstract}

Keywords: Boiling; Volume of Fluid; OpenFOAM; Contact angle; Nanofluids.

\section{Introduction}

Nowadays, the need for dissipate high heat loads is common in most industries, such as microprocessors, power generators, thermal management and transportation. One solution for this problem is the use of systems combining evaporator, condenser and electrowetting heat pipes [1]. Conventional evaporators can limit the operation of the system if they are limited by heat conduction in a film near the wall surface. The contact angle is an important factor on bubble formation and electrowetting has been proposed to modify the contact angle and enhanced boiling.

In this work, a numerical simulation of the effect of contact angle in boiling is presented. The two-phase flow is described by the VOF method. Integrated heat transport of both phases is solved. Bubble shape and temperature fields during bubble formation are analysed.

\section{Methods}

\section{1. Domain}

Figure 1 shows the schematic representation of the evaporator. A constant heat flux is imposed through a copper plaque and heats the wick imbibed with liquid. A liquid film is maintained with a constant thickness since it is continuously supplied with liquid flowing though the EHP from the condenser. A voltage difference between the copper block and liquid film surface is applied.

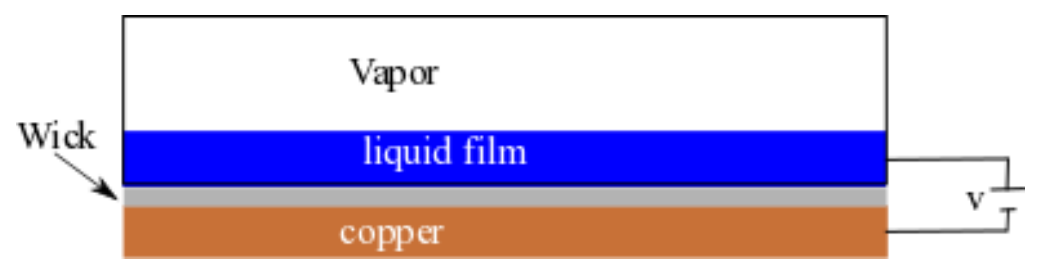

Fig. 1: Scheme of the boiler. 


\section{1. Theory}

The numerical method is implemented in OpenFoam and based on the interThermalPhaseChangeFoam solver and the work of Nabil, Rattner and Garimella[2, 3]. To solve the boiling flow, a one fluid method was adopted. The interface is tracked with the Volume of Fluid (VOF) method.

The continuity equation is given by:

$$
\nabla \cdot \vec{V}=\dot{v}_{p c}
$$

where $\vec{V}$ the velocity vector and $\dot{v}_{p c}$ is volume flow rate due to phase change.

The momentum equation is given by:

$$
\frac{\partial(\rho \vec{V})}{\partial t}+\nabla \cdot(\rho \vec{V} \vec{V})=-\nabla P+\nabla \cdot\left[\mu\left(\nabla \vec{V}+\nabla \vec{V}^{T}\right)\right]+\rho \vec{g}+\overrightarrow{f_{\sigma}}
$$

Where $\rho$ is the density of the fluid, $t$ is time, $P$ is the pressure, $\mu$ the dynamic viscosity of the fluid, $\vec{g}$ is the acceleration of gravity and $\overrightarrow{f_{\sigma}}$ represents the surface tension force term.

The surface tension force is calculated by the continuum surface force model (CSF) [4]:

$$
\overrightarrow{f_{\sigma}}=\sigma \kappa \nabla \alpha
$$

where $\sigma$ is the surface tension, $\kappa$ is the interface curvature and $\alpha$ is the phase indicator. The phase indicator represents the liquid volume fraction at a given point, with a vector position $\vec{x}$, is defined as follows:

$$
\alpha(\vec{x}, t)=\left\{\begin{array}{cc}
0 & \text { gas phase } \\
0<\alpha<1 & \text { interface } \\
1 & \text { liquid phase }
\end{array}\right.
$$

The local mean interface curvature $\kappa$ is defined as:

$$
\kappa=-\nabla \cdot \vec{n}
$$

where $\vec{n}$ is the unit vector normal to the interface:

$$
\vec{n}=\frac{\nabla \alpha}{|\nabla \alpha|}
$$

Heat transport is described by the following equation:

$$
\frac{\partial \mathrm{T}}{\partial t}+\nabla \cdot(\vec{V} T)=\nabla \cdot\left(k_{e f f} \nabla \mathrm{T}\right)-\dot{q}_{p c}
$$

where $\mathrm{T}$ is temperature, $k_{e f f}$ is the effective heat transfer coefficient and $\dot{q}_{p c}$ is the heat generated by phase change.

The transport equation for the phase indicator is: 


$$
\frac{\partial \alpha}{\partial t}+\vec{V} \cdot \nabla \alpha+\nabla \cdot\left[\alpha(1-\alpha) \vec{V}_{c}\right]=\dot{\alpha}_{p c}
$$

where $\dot{\alpha}_{p c}$ is the phase generation due to phase change and $\vec{V}_{c}$ is the compressive velocity:

$$
\vec{V}_{c}=\min \left\{C_{\alpha}|\vec{V}|, \max (|\vec{V}|)\right\} \vec{n}
$$

Here $C_{\alpha}$ is the compressibility factor.

The viscosity, density and heat transfer coefficient are calculated by:

$$
\begin{gathered}
\mu=\alpha \mu_{L}+(1-\alpha) \mu_{G} \\
\rho=\alpha \rho_{L}+(1-\alpha) \rho_{G} \\
k_{e f f}=\alpha k_{e f f_{L}}+(1-\alpha) k_{e f f_{G}}
\end{gathered}
$$

Here $L$ and $G$ refer to liquid and gas phases, respectively. The values of $\dot{\alpha}_{p c}$ and $\dot{v}_{p c}$ are given by:

$$
\begin{gathered}
\dot{\alpha}_{p c}=\frac{\dot{q}_{p c}}{i_{\mathrm{LV}}}\left(\frac{1}{\rho_{V}}-\frac{1}{\rho_{L}}\right) \\
\dot{v}_{p c}=-\frac{\dot{q}_{p c}}{\rho i_{\mathrm{LV}}}
\end{gathered}
$$

where $i_{\mathrm{LV}}$ is the phase change enthalpy.

The heat generated by phase change is calculated by following the Interface Equilibrium Split Dilation model [2] based on the work of Rattner [5].

To determine the electrostatic effect on bubble formation, the electric field is calculated by solving the Gauss equation. The interaction between the electric field and the contact angle of the bubbles formed is given by the Young-Lippmann equation.

\section{Results}

Bubble formations was studied for several contact angles in the central region of the boiler. Figure 2 shows the results for a water vapor bubble formation for a constant heat flux $\left(1900 \mathrm{~W} / \mathrm{m}^{2}\right)$ and a contact angle of $159^{\circ}$.

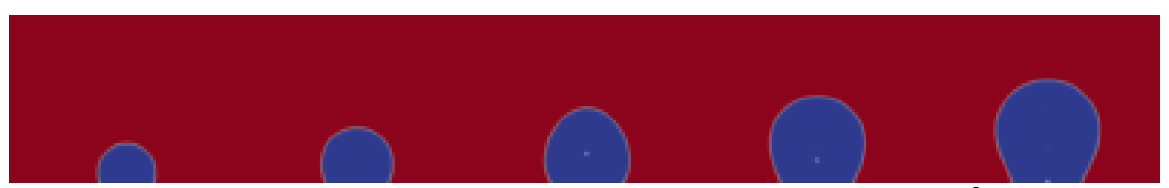

Fig. 2: Evolution of the bubble formation. Results for a constant heat flux $\left(1900 \mathrm{~W} / \mathrm{m}^{2}\right)$ and a contact angle of $159^{\circ}$.

Work is under way to study the contact angle and electrostatic effects on bubble formation. Different fluids will also be considered, including new nanofluids.

\section{Conclusion}

Bubble formation in a boiler was studied by numerical methods. The solver interThermalPhaseChangeFoam from OpenFOAM was used to solve the flow, heat transport and phase indicator equations. Preliminary results show that numerical methods can be used to study the formation of water vapor bubbles during boiling. Further work is required to study the effect of contact angle and the operation of the boiler under electrostatic effects. The numerical results will be validated by experimental data. 


\section{Acknowledgements}

The authors acknowledge to Fundação para a Ciência e a Tecnologia (FCT) for partially financing the research under the framework of the project UTAP-EXPL/CTE/0064/2017, under 5665 - International Partnerships in Science and Technology, UT Austin Programme. A.S. Moita also acknowledges FCT for financing her contract through the IF 2015 recruitment program (IF 00810-2015) and through the exploratory project associated with this contract.

\section{References}

[1] R. S. Hale and V. Bahadur, "Electrowetting heat pipes for heat transport over extended distances, " IEEE Transactions on Components, Packaging and Manufacturing Technology, vol. 5, no. 10, pp. 1441-1450, 2015.

[2] M. Nabil and A.S. Rattner, "interThermalPhaseChangeFoam-A framework for two-phase flow simulations with thermally driven phase change," SoftwareX, vol. 5, pp. 216-226, 2016.

[3] A. S Rattner and S. Garimella, "Simple mechanistically consistent formulation for volume-of-fluid based computations of condensing flows," Journal of Heat Transfer, vol. 136, no. 7, pp. 071501, 2014.

[4] J. U. Brackbill, D. B. Kothe, and C. Zemach, "A continuum method for modeling surface tension," Journal of Computational Physics, vol. 100, no. 2, pp. 335-354, 1992.

[5] A. S. Rattner, "Single-pressure absorption refrigeration systems for low-source-temperature applications," Georgia Institute of Technology, 2015. 